\title{
Co-Existing Periodic Beaconing and Hazard Warnings in IEEE 802.11p-Based Platooning Applications
}

\author{
Annette Böhm, Magnus Jonsson and Elisabeth Uhlemann \\ CERES (Centre for Research on Embedded Systems) \\ Halmstad University, Halmstad, Sweden \\ \{Annette.Bohm, Magnus.Jonsson, Elisabeth.Uhlemann\}@hh.se
}

\begin{abstract}
A platoon of trucks driving at the same, mutually agreed speed while keeping a minimum inter-vehicle distance will reduce fuel consumption, enhance transport efficiency as well as improve the safety of other adjacent road users. The European profile of IEEE 802.11p for inter-vehicle communications uses a single $10 \mathrm{MHz}$ control channel dedicated to safety-critical data, shared by periodic status updates, CAM (Cooperative Awareness Message), and event-triggered warnings, DENM (Decentralized Environmental Notification Message). Coupled with the random access delay inherent to the 802.11p medium access method, the strict timing and reliability requirements of platoon applications are not easily met. To this end, we evaluate by simulation the effect of IEEE 802.11p-compliant send rate adaptations and message type prioritizations and the choice of warning dissemination strategy on CAM transmissions and DENM dissemination in a platooning scenario. Simulation studies of a platoon of 10-20 vehicles in a busy highway scenario show that the context-aware choice of send rate, priority class and dissemination strategy not only reduce the dissemination delay of DENMs but even has a significant effect on the throughput of CAMs exchanged by platoon members.
\end{abstract}

\section{Keywords}

ITS, VANET, platooning, IEEE 802.11p, CAM, DENM.

\section{INTRODUCTION}

Cooperative Intelligent Transport Systems (ITS) have the potential to considerably improve the level of safety, comfort and efficiency on our roads. Many emerging cooperative traffic safety applications are based on the periodic exchange of status messages between vehicles, while others rely on the fast dissemination of warning messages triggered by an unforeseen event, e.g. a driver suddenly breaking or attempting an unexpected lane change. An application area where the gain from inter-vehicle communications is particularly obvious and advantageous is platooning. A platoon of trucks driving at the same, mutually agreed speed while keeping a minimum inter-vehicle distance will reduce fuel consumption [1], enhance transport efficiency and improve the safety of other adjacent road users.

The strict timing and reliability requirements of such platoon applications are not easily met. Consider the recently adopted IEEE 802.11p standard [2] for short-range inter-vehicle communications: it uses a random access protocol for medium access control (MAC), coupled with the European requirement to use only one $10 \mathrm{MHz}$ control channel dedicated to the exchange of safety-critical ITS related data. This control channel is shared by two message types, CAMs (Cooperative Awareness Messages) and DENMs (Decentralized Environmental Notification Messages), where the former refers to periodically exchanged status updates while the latter comprises all types of eventtriggered warning messages. If the control channel is heavily loaded with data traffic, the random delays inherent to the IEEE 802.11p MAC method may prevent proper functionality of a platooning application, or alternatively, the required safety distance between members in a platoon may be too long to enable reduced fuel consumptions. The strict timing and reliability requirements of the platooning application stem from the fact that it is a distributed control application relying on the timely dissemination of hazard warnings and periodic status messages. If a platooning application based on IEEE 802.11p is to be realized, it is thus important to reduce the dissemination delay for warning messages and other types of critical events. At the same time, periodic status update messages or beacons will be required both by the platooning application itself and by other coexisting applications. A vehicle that is not able to successfully transmit a CAM to during two or more CAM periods due to an overloaded channel remains invisible to its neighbors during that time span. Instead of being integrated into the CAM-based safety application it now becomes a safety hazard. Hence, acceptable service to other message types must be maintained even in the presence of eventtriggered warning messages.

To increase the safety and efficiency of platooning applications, we investigate the effect of the following parameters on both DENM dissemination and CAM throughput in a platooning scenario: the choice of IEEE 802.11p priority setting for the two data traffic classes, the choice of send rate for DENMs and the dissemination strategy used to spread these event-triggered warning messages to all platoon members. All the suggested and evaluated schemes and parameter settings are in compliance with the IEEE 802.11p standard. Our simulation study shows that the choice of dissemination scheme not only has the potential to reduce the dissemination delay of hazard warnings but even considerably improves the throughput of periodically exchanged CAM traffic. We further evaluate the effect of different combinations of the IEEE 802.11p-based priority settings and send rates on the CAM and DENM message types. Parameters that influence these choices are e.g. the number of platoon members, the overall radio conditions or the number of surrounding vehicles the platoon shares the common control channel with. For a platoon of 20 vehicles with $30 \mathrm{~m}$ antenna-toantenna spacing and the assumption of a busy highway with heavy overall channel utilization, we were able to achieve a platoon warning time of $6 \mathrm{~ms}$ or less for $80 \%$ of the simulated test runs while the entire platoon received a warning in $100 \%$ of the test 
runs after $10 \mathrm{~ms}$ or less while a reasonable CAM update frequency could be maintained. To the best of our knowledge, this combination of reducing the dissemination delay of hazard warnings while at the same time providing enough resources to support the frequent exchange of periodic status messages has not been considered previously. Our study therefore provides valuable input to the parameter selection for time-critical applications based on the co-existence of the CAM and DENM traffic types on a common data channel, both for platooning applications in particular and other cooperative traffic safety applications in general.

\section{PREREQUISITES FROM STANDARDI- ZATION}

The recent allocation of dedicated frequency channels for intervehicle communication and the introduction of IEEE 802.11p [2], constitute the first step towards future proactive, cooperative ITS applications. The European Telecommunications Standards Institute (ETSI) has standardized a profile of IEEE 802.11p adapted to the $30 \mathrm{MHz}$ frequency spectrum at the $5.9 \mathrm{GHz}$ band allocated in Europe [3]. ETSI considers two types of messages, Cooperative Awareness Messages (CAM) [4] and Decentralized Environmental Notification Messages (DENM) [5]. CAMs are broadcasted periodically, typically at a rate of $2-10 \mathrm{~Hz}$, stating vehicle speed, position and driving direction, whereas DENMs are event-driven and application specific warning messages. One dedicated control channel is reserved for and shared by CAMs and DENMs. In the platooning case, DENMs would be used to issue warnings, spread notification and control information within the platoon, both in the rare event of an emergency and on a more regular basis to control e.g. the speed and setup of the platoon. The periodic CAMs are always present and form the basis for both platoon-related and non-platoon-related ITS applications.

The MAC layer of IEEE 802.11p uses CSMA/CA, where a node only attempts to transmit if the channel is sensed free during a short time period (Arbitration Inter Frame Spacing, AIFS). If the channel is busy or if it becomes busy during the AIFS, the node randomizes a backoff time, which is counted down only during time periods when the channel is sensed free. When the backoff value reaches zero, the node transmits directly without any further delay. Since messages are broadcasted, no acknowledgements are used and thereby no collision detection is possible. Therefore, the contention window is never extended as in traditional IEEE 802.11 and maximum one backoff procedure is invoked. IEEE 802.11p defines four priority levels characterized by the length of the AIFS and the parameters of the backoff window. This way, DENMs can be given prioritized channel access over CAMs. Due to the random nature of the 802.11 p MAC protocol, a channel utilization level (Channel Busy Time, CBT) of $25 \%$ or less is recommended by ongoing ETSI standardization [3].

\section{SELECTING SUITABLE PARAMETERS AND SETTINGS}

The overall goal of this work is to reduce the DENM dissemination delay within a platoon, while at the same time maintaining acceptable service to other message types like CAM. To this end investigate the effect of three parameter choices explained in detail below:
1. The selection of the IEEE 802.11p priority levels for CAM and DENM transmissions.

\section{Evaluate different DENM send rates}

3. Evaluate a platoon specific dissemination model that prioritizes fast re-broadcast of DENMs backwards within the platoon

\subsection{IEEE 802.11p Priority Class}

The IEEE 802.11p standard defines four different priority classes to support traffic with different quality of service requirements. However, the preferred choice of priority level for the message types CAM and DENM when sent on the common control channel is not yet specified.

The distinction between priority classes for IEEE 802.11p is achieved by different AIFS durations and different contention window sizes. See Table 1. Each node has to listen to the channel for the specified AIFS duration determined by its priority class. Only if the channel remains idle during this listening period, the node is allowed to start its transmission. Consequently, a node carrying data of a higher priority class can potentially outcompete a node with data in a lower class during the AIFS waiting time. A node that does not gain access to the channel starts a backoff timer that is only counted down while the channel is free. Once the timer reaches zero, the packet is transmitted. The start value of the backoff timer is chosen randomly from a given number of values. Priority level 1 has for example three different values to choose from $(1 *$ slot_time, $2 *$ slot_time and $3 *$ slot_time, where a slot time is $13 \mu \mathrm{s})$, level 2 has seven selectable values etc. It should be noted that fewer backoff values leads to a higher probability of more than one node selecting the same backoff value, leading to simultaneous transmission attempts once the timer expires.

In [6], Reinders et al. study the impact of the contention window sizes defined in IEEE 802.11p on the delay and inter-arrival time of periodically broadcasted status messages. Although timecritical ITS safety applications are the target, only the average CAM inter-arrival time is studied, while our evaluation provide the worst-case inter-arrival time, producing more significant results for a real-time application. Furthermore, the authors of [6] assume a channel with CAM traffic only and therefore do not investigate the effect that different combinations of priority settings for both periodic and event-based traffic has on either of those message types.

Table 1. Settings for IEEE 802.11p priority classes

\begin{tabular}{|c|c|c|c|}
\hline Priority & $\boldsymbol{C W m i n}$ & $\boldsymbol{C W m a x}$ & $\boldsymbol{T}_{\text {AIFS }}[\boldsymbol{\mu s}]$ \\
\hline Class 1 & 3 & 17 & 58 \\
\hline Class 2 & 7 & 15 & 71 \\
\hline Class 3 & 15 & 1023 & 110 \\
\hline Class 4 & 15 & 1023 & 149 \\
\hline
\end{tabular}

\subsection{DENM Send Rate}

Due to the broadcast nature of CAMs and DENMs, no acknowledgements are sent and a vehicle is therefore unable to determine if its packet has been successfully received. This implies that DENMs would have to be repeated periodically until the entire platoon can be expected to have received the warning. 
According to ETSI, a DENM send rate of 1-20 Hz should be used for such repetitions. Depending on the nature of the event, the relative speed of the vehicles, the number of platoon members and the current channel load, the DENM send rate could potentially be increased beyond the suggested $20 \mathrm{~Hz}$, provided that acceptable service still can be given to CAMs. To this end, we evaluate two different DENM rates: $10 \mathrm{~Hz}$ and $100 \mathrm{~Hz}$.

Improved service for safety messages in ITS applications has been considered previously, in e.g. [6-7]. While [6] uses the distance to a potential collision area as input parameter to an adaptive, context aware transmission policy, in [7,] the CAM send rates are adapted according to the role of each vehicle in an overtaking warning application. However, no previous papers have evaluated the performance of the two messages types jointly such that the improvement of hazard warnings cannot be done at the expense of periodic updates.

\subsection{Dissemination Strategy}

As soon as the distance between the platoon leader and a platoon member exceeds the achievable radio range at a given point in time, a dissemination strategy is needed, allowing intermediate vehicles who successfully received the DENM transmission by the platoon leader to relay this information to the back of the platoon. In the absence of acknowledgements, one important parameter is the number of times a vehicle should periodically repeat a DENM after its initial reception. Knowledge of the current radio conditions, platoon length and network load could provide input to the suitable choice of this number. Simple flooding by letting each vehicle repeat a DENM periodically $x$ times, can provide a tradeoff between the need to maintain a low delay for a successful platoon warning and the goal to reduce the overall network load to not hinder other types of messages.

A DENM that originates from the platoon leader reaches members of the platoon that are within radio range at approximately the same time. If all platoon members that successfully receive this DENM also re-broadcast, the first round of re-broadcasts will happen within a very short time window (somewhat depending on the propagation delay, the total number of successful DENM receivers and, amongst those, the number of nodes that are forced into backoff due to an busy channel). Although it is our main goal to decrease the DENM dissemination delay within the platoon, it is important to keep in mind that the control channel is shared by all vehicles and message types. The success of both CAMs and DENMs is highly dependent on the overall channel load. If a channel is utilized heavily enough to not give a packet in backoff mode the chance to count down its backoff timer and start its transmission before a certain deadline, packets are dropped. For periodic traffic, the generation of a new packet can be seen as the point in time (or deadline) when a packet expires. The more packets that are forced into backoff, the higher the probability that two or more packets try to access the channel simultaneously with packet collisions as a consequence. It is therefore desirable to keep the amount of data traffic involved in the platoon warning process to a minimum. To this end we suggest the following adaptive flooding models.

\section{a) Flooding with $x$ repetitions}

Upon reception of its first warning, a vehicle starts rebroadcasting DENMs periodically. After a predefined number of repetitions, the re-broadcasting is stopped. Flooding with 5 repetitions in a platoon of 20 vehicles implies that $20 \times 5$ DENMs in total will have been transmitted until the dissemination process seizes. This number could be continuously adapted to changes in platoon length, current radio conditions or channel busy time.

\section{b) Flooding with $x$ repetitions until DENM reception from behind}

As we consider a DENM spreading from the leading vehicle backwards as the most common scenario in platooning, we suggest that a vehicle seizes its re-broadcasts as soon as it receives a DENM from a vehicle situated further back in the platoon. The vehicle sees this as an indication that the warning dissemination has successfully progressed backwards in the platoon and will continue doing so without its help. (Note that all platoon members know their respective position in the platoon.) Depending on how quickly warnings are spread to the back of the platoon, there will be vehicles that only re-broadcast a DENM once or will not take part in the dissemination process at all, leading to a significant reduction in overall channel load. This dissemination strategy allows for a generous number of repetitions without automatically loading the channel with unnecessary DENM transmission, while still maintaining a high probability of a successful platoon warning even in highly variable radio environments.

In [9], Bür and Kihl compare several selective DENM broadcast algorithms for safety applications in Vehicular Ad-Hoc Networks (VANETs), amongst others intelligent flooding that seizes after overhearing re-broadcasts. The authors measure the effect of those strategies on the DENM dissemination process, e.g. on the warning time of the last reachable vehicle in a platoon, but do not consider their impact on other messages types like co-existing CAMs broadcasted within the platoon.

\section{SIMULATION RESULTS}

To evaluate the performance of different priority and send rate settings, as well as the proposed dissemination strategies, a computer simulation study was conducted in Matlab.

\subsection{Simulation Details}

The goal of this simulation study is to investigate the effect our choice of parameters has both on the periodic CAM traffic and the event-based DENM dissemination. We consider a busy highway scenario and a network consisting of a platoon (10 or 20 platoon members) and up to 100 surrounding vehicles that are not part of the platoon. The results are based on 500 simulated runs per case, where one run corresponds to one simulated second and one case corresponds to a specific combination of priority settings, send rates and dissemination models. During one run, each vehicle sends out CAMs periodically whereas DENMs are only sent by platoon members. The DENM dissemination process is triggered by the platoon leader and is caused by an imaginary event occurring after each vehicle has transmitted at least one CAM. Following the ETSI TC ITS standard, we use a packet size of 400 byte and a data rate of $6 \mathrm{Mbit} / \mathrm{s}$. The AIFS and backoff window sizes are based on a slot time of $13 \mu \mathrm{s}$. The parameters common to all simulated cases can be found in Table 2 .

Consider a platoon of tightly spaced vehicles driving on a busy highway. We assume that the leading vehicle has a coordinating role in the platoon, such that DENMs originate from the leading vehicle for dissemination backwards in the platoon. As it cannot be presumed that all platoon members are within radio range of each other, DENMs need to be relayed or re-broadcasted either periodically or upon reception. We consider a platoon of 10-20 vehicles with a spacing of $30 \mathrm{~m}$ (antenna to antenna) and a radio 
range of approximately $500 \mathrm{~m}$. This implies that, in the case of 15 platoon members or more, DENMs have to be relayed/rebroadcasted to reach all members of the platoon. The choice of both radio range and line-of-sight (LOS) channel model is based on results from field trials conducted in [10]. Further, we model shadowing by other vehicles (obstructed LOS) using Rayleigh fading in addition to the LOS model in [10]. When a vehicle is located in-between a sender and a receiver, we reduce the probability of LOS to $20 \%$. For each additional vehicle that is located in-between the sender and the receiver, we reduce the probability another $5 \%$, implying that when five vehicles are located in-between a sender and a receiver, the probability of LOS is zero and thereby Rayleigh fading is used in combination with the LOS model.

Table 2. Simulation parameters

\begin{tabular}{|l|l|}
\hline Packet size (CAM, DENM) & 400 byte \\
\hline Data rate & $6 \mathrm{Mb} / \mathrm{s}$ \\
\hline Slot time & $13 \mu \mathrm{s}$ \\
\hline $\begin{array}{l}\text { Antenna-to-antenna spacing } \\
\text { (platoon only) }\end{array}$ & $30 \mathrm{~m}$ \\
\hline Transmission range & $500 \mathrm{~m}$ \\
\hline \# platoon vehicles & 10,20 \\
\hline \# extra vehicles & 10,100 \\
\hline CAM send rate & $10 \mathrm{~Hz}$ \\
\hline DENM send rate & $10,100 \mathrm{~Hz}$ \\
\hline
\end{tabular}

To model a busy highway situation with data traffic from additional co-existing applications, we assume that the platoon has a set of non-platoon-related vehicles (so-called extra vehicles) within radio range of all platoon members. To evaluate the performance under different network loads we consider two cases: 10 or 100 extra vehicles. Both platoon members and extra vehicles send out periodic CAMs while DENMs are only generated by platoon members. Only data traffic within the platoon are monitored and used for our evaluation, whereas extra vehicles mainly serve as a way to tune the overall load on the common channel. Although platoon lengths of both 10 and 20 vehicles were simulated, only results for the 20 vehicle cases are presented here as the results are similar, but the trends are clearer for a longer platoon.

The performance is measured using two different performance metrics: a) the DENM dissemination delay and b) the CAM up-todateness.

\section{a) DENM dissemination delay}

The hard timing requirements of a safetly-critical platooning application can only be met if the entire platoon is made aware of an unexpected event within a tight time frame. The DENM dissemination delay is defined as the delay from the generation of the first DENM by the platoon leader (caused by an imaginary event) until every platoon member has received a warning about this particular event, i.e. until the point in time when the DENM dissemination process is concluded. Each simulated run produces one value for the DENM dissemination delay (DD). In our evaluation, the results are presented as a cumulative distribution function (CDF) of the DENM DD averaged over 500 runs.

\section{b) CAM up-to-dateness}

During an on-going DENM dissemination, CAMs have to share the common bandwidth with the event-triggered warning messages. Many applications, including platooning applications, rely on the availability of up-to-date status information exchanged through CAMs. In order to make sure that a certain level of CAM throughput is maintained despite the presence of DENMs in the network, we monitor the freshness, or up-to-dateness of the CAMs. The CAM up-to-dateness (UTD) can be considered as the packet inter-arrival time, i.e., the time elapsed between when a particular receiver gets a CAM from a particular sender, to when it gets the next one from the same sender. For simplicity, we only monitor the CAMs generated by platoon members and recevied from the neighbor in-front, as we assume that this neighbor is one of the most important to keep track of. In a network with low traffic load, the CAM UTD is around one CAM period on average, e.g. $0.1 \mathrm{~s}$ for a CAM send rate of $10 \mathrm{~Hz}$, as a new CAM with fresh information is generated every $0.1 \mathrm{~s}$. However, the interesting parameter to study is the worst-case CAM UTD for a simulated run, i.e. the longest period of time during which a vehicle did not receive a new CAM (from a specific vehicle). This value corresponds to the worst case time a vehicle is "invisible" to its successor. Each simulated run produces one worst-case CAM UTD time for every platoon member (except the platoon leader who has no in-front neightbor) which is used to determine the average worst-case CAM UTD for the entire run. Also in this case, the results are presented as the CDF of the worst case CAM UTD, average over all platoon members and over 500 simulated runs.

\subsection{Simulation Evaluation}

Figures 1 to 4 evaluate the impact of different combinations of priority levels on the DENM DD and the CAM UTD. Fixed parameters for these cases are: a platoon length of 20 vehicles, a CAM and DENM send rate of $10 \mathrm{~Hz}$ and the dissemination method of flooding with 5 repetitions. In figures 1 and 2, there are 10 extra vehicles in addition to the platoon members, whilst this number is increased to 100 in Figures 3 and 4. AIFS length and contention window size are chosen according to the class 1-3 settings of the IEEE 802.11p standard, Table 1.

In Figure 1 and 2 we can see the DENM DD and the CAM UTD for five different priority combinations in a network with 10 extra vehicles. We consider the cases when both CAM and DENM have the same priority, either both messages types have priority class 1 or both have priority 2 . We also consider three cases where CAM and DENM have different priorities, such that DENM has priority 1 and CAM has priority 1, 2, and 3 respectively. As the contention window size for priority class 4 is equal to the one used in class 3 , class 4 was not considered for simulation. Figures 1 show that, in the presence of 10 extra vehicles, the choice of priority settings does not considerably influence the DENM dissemination delay in the platoon. The total network load is low enough to give packets of both message types access to the shared medium without delays that significantly favor a certain combination of priority classes. For all simulated runs, the entire platoon received a warning within $10 \mathrm{~ms}$ from event detection, and for $85 \%$ of the runs, the DENM dissemination delay was even below 6 ms (with an exception of the case where DENMs did not have the highest priority which performed slightly worse). Figure 2 reveals a similar situation with a CAM UTD for all priority combinations. In $60-65 \%$ of the simulated runs, the worst case CAM UTD 


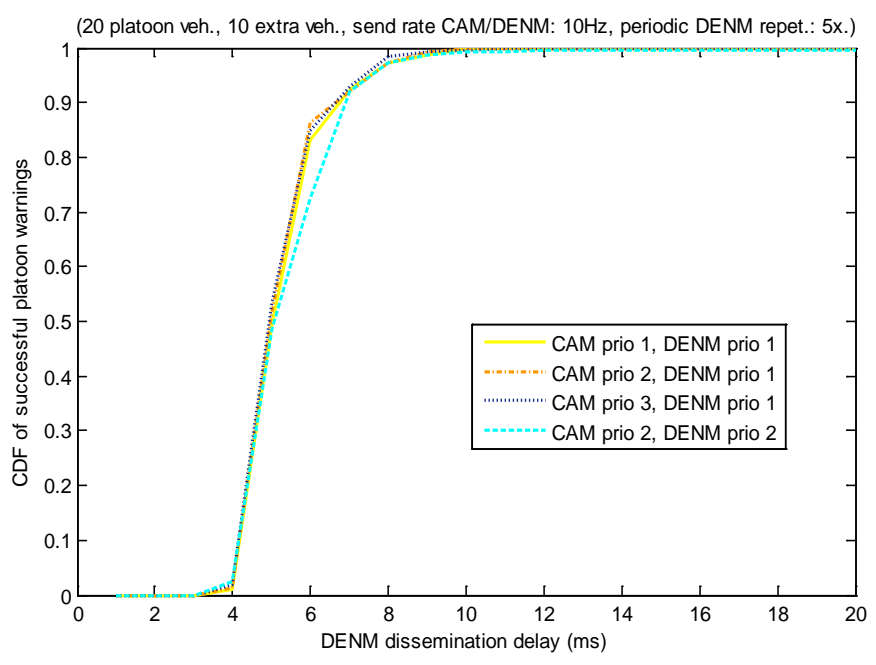

Figure 1: DENM delay for varying priority classes (10 extra veh.)

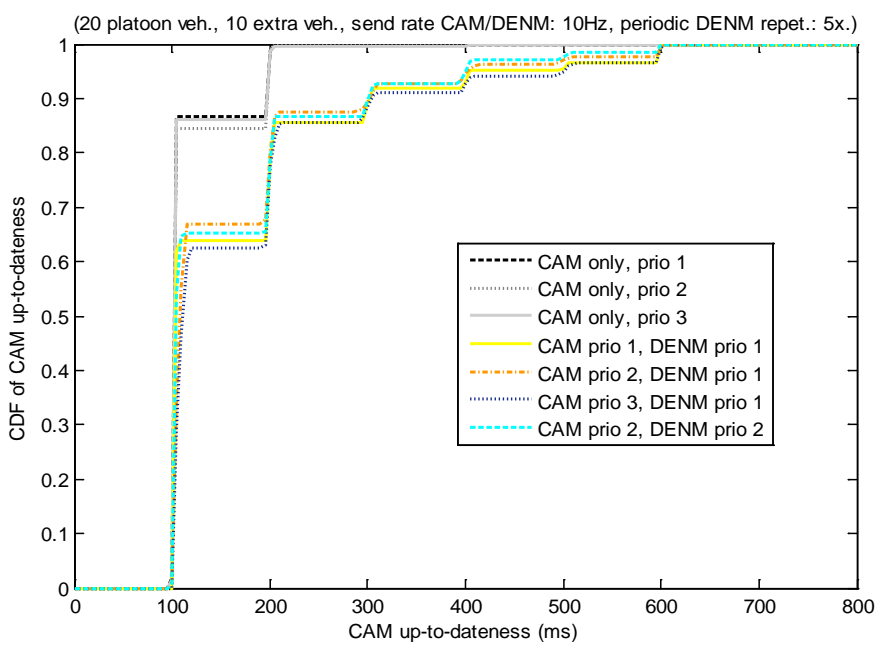

Figure 2: CAM up-to-dateness for varying priority classes (10 extra veh.)

remained in the range of one CAM period, while only 2-3 \% cases show a worst-case delay of $500 \mathrm{~ms}$ until a new updated CAM arrived, which implies that the vehicle in front was "invisible" for $0.5 \mathrm{~s}$. In Figure 2, also the results for cases with only CAM traffic, i.e., no DENM were issued, are shown as a benchmark. It can be concluded that, even in a lightly loaded network, the CAM UTD is influenced by the presence of event-triggered DENM traffic.

Results with a heavily loaded network, where resources are shared between a platoon with 20 members and 100 extra ve-hicles, are shown in Figures 2-3. Here, the importance of a proper choice of CAM and DENM priority levels is clearly visible. A significant increase in DENM DD for shared priority classes, i.e., when both CAM and DENM have the same priority, can be observed, leading to a clear distinction be-tween cases with "same priority" and "different priority". Worst-case DENM DDs of up to 320ms were recorded for the cases of shared priority whereas a platoon warning was achieved within $10 \mathrm{~ms}$ by all simulated test runs when different priorty levels for CAM and DENM were chosen. As long as CAMs have a lower priority, DENMs will always get

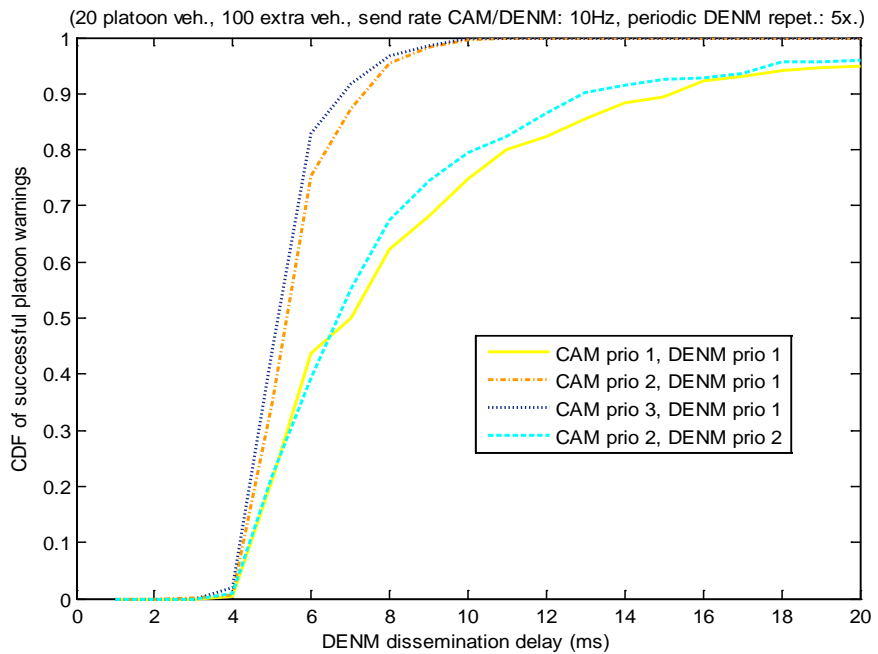

Figure 3: DENM delay for varying priority classes (100 extra veh.)

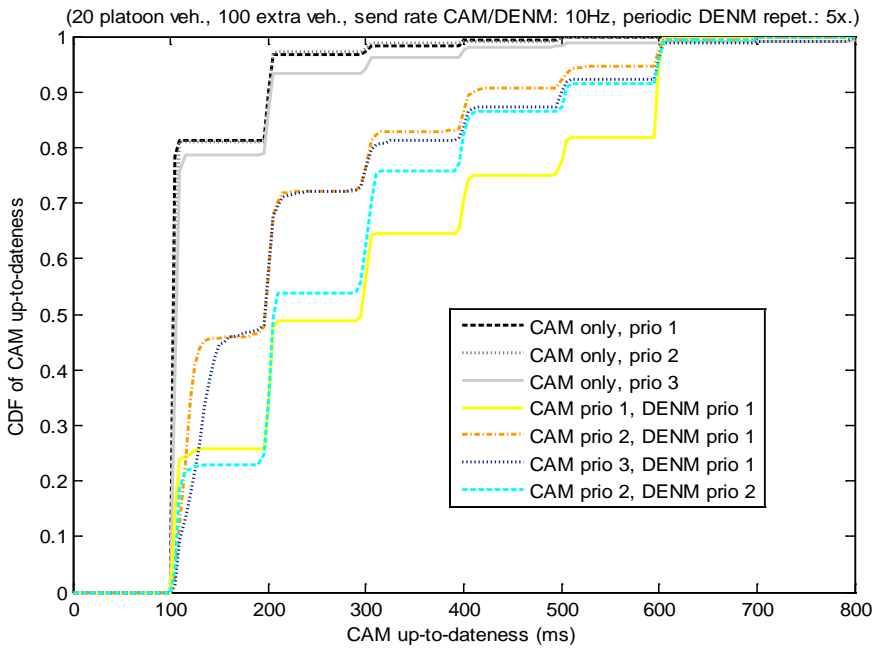

Figure 4: CAM up-to-dateness for varying priority classes (100 extra veh.)

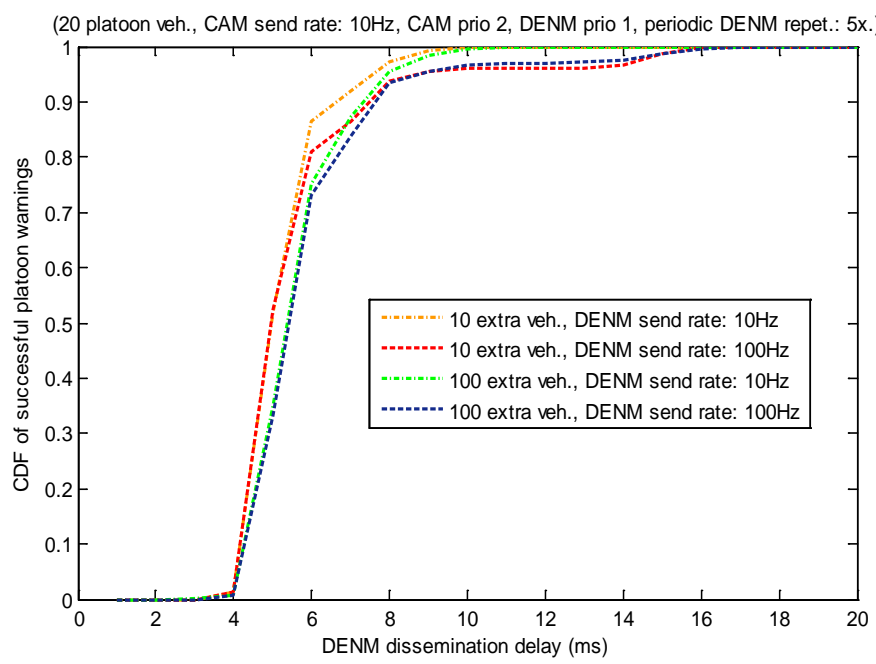

Figure. 5: DENM delay for varying send rates 


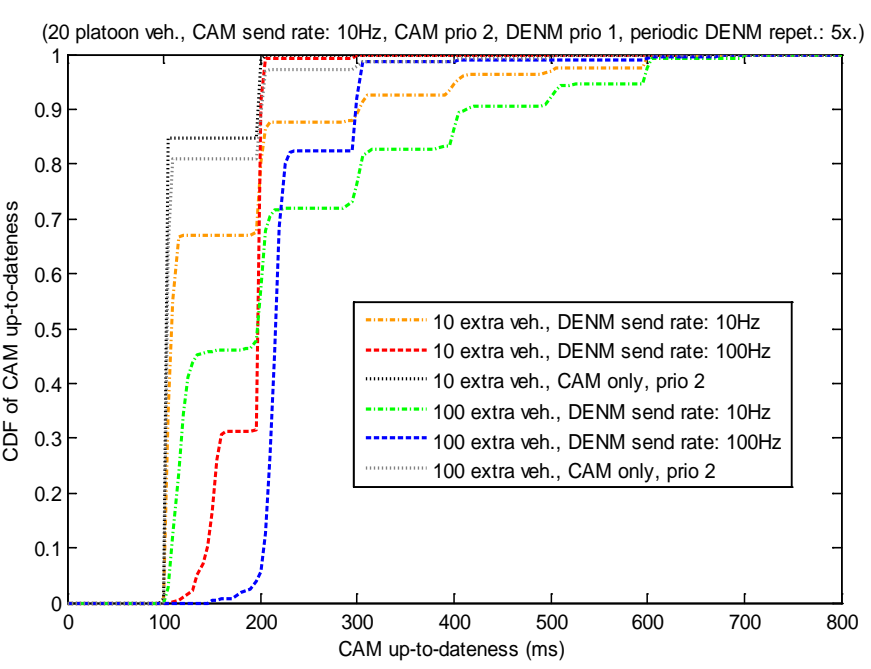

Figure 6: CAM UTD for varying send rates

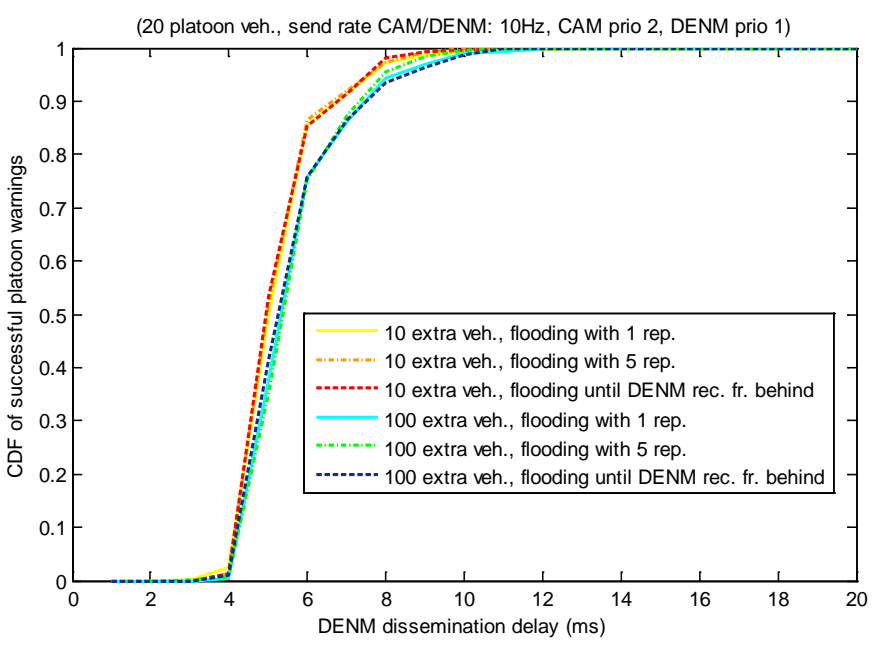

Figure 7: DENM delay for varying dissemination models

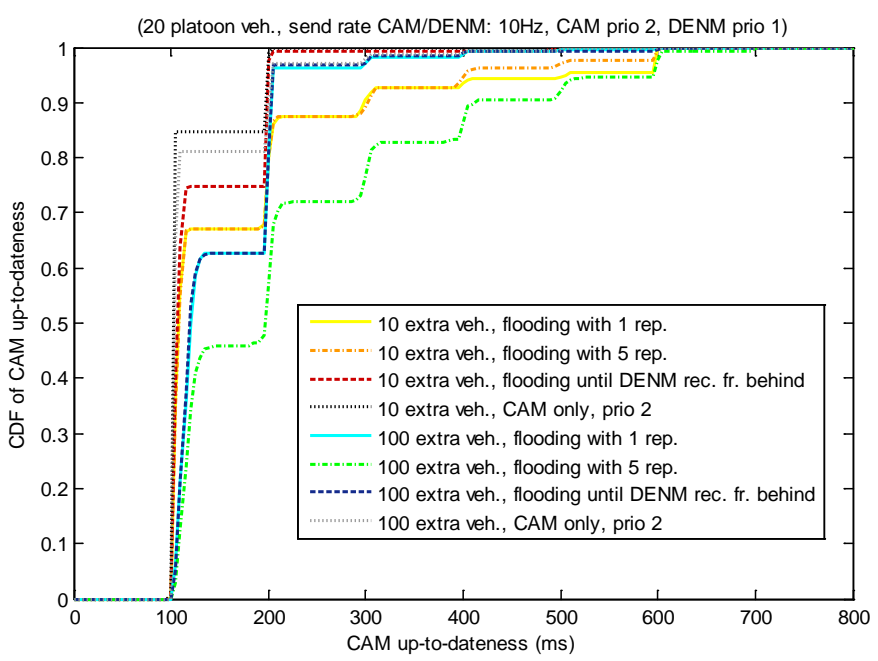

Figure 8: CAM UTD for varying dissemination models access to the channel first due to lower AIFS values. Also in case of a backoff, a DENM has a higher probability to choose a smaller value for its backoff timer, which leads to quicker channel access. To have both CAM and DENM at the same priority level leads to decreased performance both in terms of DENM delay and CAM UTD. This is due to the fact that both message types now compete for the medium with the same parameter settings, increasing the chance of simultaneous transmission attempts and packet collisions. The slight advantage of the case where both message types share priority level 2 over the case where both share priority level 1 can be explained by fewer contention window values offered by priority 1 , resulting in a higher probability that nodes end up accessing the channel simultaneously after a backoff phase. This impacts both message types and it can be concluded that lowering the CAM priority from 1 to 2 or even 3 actually has a positive effect on the CAM UTD even if DENMs have higher priority. In cases where CAM and DENM have different priority, a worst-case CAM UTD of around $200 \mathrm{~ms}$ can be seen for $70 \%$ of the simulated runs, i.e. two CAM periods, whereas this number only was achieved by approx. $50 \%$ of the runs in the cases where both messages types have the same priority. The slightly improved DENM dissemination delay for CAM priority class 3 over priority 2 can be explained by the longer AIFS imposed on a CAM packet of priority class 3 , giving DENMs a greater chance gain access to the channel before a CAM.. From the comparison of different priority classes, we conclude that the combination CAM class 2 and DENM class 1 is the most favorable one, not only in terms of DENM DD, but also, in CAM UTD. It is noteworthy that the performance improvement is not a trade-off between the two measures CAM UTD and DENM DD, but it is actually the best settings for both message types. Consequently, we keep these priority settings fixed for the rest of the evaluation.

Figures 5 and 6 show the impact of different DENM send rates on the DENM DD and the CAM UTD, respectively. Intuitively, an increased DENM send rate from $10 \mathrm{~Hz}$ to $100 \mathrm{~Hz}$ should lead to a reduction in the DENM DD. As can be seen in Figure 5, however, the higher send rate of $100 \mathrm{~Hz}$ actually results in a longer DENM DD. Since a full platoon warning is achieved within a single DENM period for a majority of all cases, the periodic generation of new DENM re-broadcasts after $10 \mathrm{~ms}$ simply slows down the dissemination process by introducing packets into an already heavily loaded network without adding much benefit. The high amount of DENMs in the system leads to a situation where DENMs that actually are useful for the dissemination process are kept from accessing the channel, resulting in longer delays before the full platoon is warned in about $4 \%$ of the simulated runs.

In Figure 6, it is very clearly visible that the choice of DENM send rate influences the CAM performance. However, it is less clear which case actually is the best when considering the CAM UTD. A high DENM send rate means a greater burst of DENM packets, but during a shorter period of time. After the burst, the channel is again available to CAMs, which can be seen by a sudden steep increase of the CDF for the cases with $100 \mathrm{~Hz}$. For a lower DENM send rate, on the other hand, the DENM dissemination process is spread out over a longer period of time, giving more CAMs a chance to access the channel even during the beginning of the DENM dissemination phase, but then resulting in a more consistent interference, that can be seen in the slower increase of the CDF for the CAM UTD for the case with $10 \mathrm{~Hz}$. For the case with 100 extra vehicles and a DENM rate of $100 \mathrm{~Hz}$, this leads to a CAM UTD of around 200ms or above for all simulated cases. This means that during the DENM dissemination phase, the channel is so heavily occupied by DENMs that it is difficult for CAMs to get channel access, resulting in a worst case CAM UTD of two CAM periods or more for all simulated runs. However, after the DENM dissemination phase, the CAM UTD 
quickly recovers in around $80 \%$ of the simulated runs. The negative effect of a high DENM send rate on the CAM UTD is confirmed by monitoring the average number of CAMs that were discarded during one run due to the arrival of a new periodic status message (not shown in a figure). This number lies around $10 \%$ for a $100 \mathrm{~Hz}$ DENM send rate while no packets had to be dropped at the lower DENM send rate of $10 \mathrm{~Hz}$. Furthermore, it can be seen that for 10 extra vehicles and $10 \mathrm{~Hz}$ DENM rate, the CDF of the CAM UTD is up to a level corresponding to not having DENMs in the system, after the initial delay of $200 \mathrm{~ms}$.

The proper choice of DENM send rate therefore depends on several factors. While a send rate of $100 \mathrm{~Hz}$ leads to DENM DD of around $15 \mathrm{~s}$, a send rate of $10 \mathrm{~Hz}$ achieves a DENM DD of 10 ms. This difference can be crucial for highly time-critical applications and thereby motivate the choice of a lower send rate. However, quite counterintuitive, a high DENM send rate can be more beneficial for the CAM UTD than a lower rate. For a DENM send rate of $10 \mathrm{~Hz}$ and 100 extra vehicles, the CAM UTD is around one CAM period for $45 \%$ of the simulated runs, while none of the simulated runs for the $100 \mathrm{~Hz}$ rate achieved a CAM UTD under 2 CAM periods. On the other hand, lower DENM send rates lead to a longer, continuous interference with CAM traffic and hereby longer worst-case CAM delays. This might be a problem for control applications relying on fresh status data provided by the exchange of CAMs, when a CAM UTD of 500$600 \mathrm{~ms}$ simply is unacceptable. For the scope of this evaluation, we choose to continue with a DENM send rate of $10 \mathrm{~Hz}$, as this gives the best trade-off between the performance for the two message types.

In the last case, we study the effect of two different dissemination models on the DENM DD, Figure 7, and the CAM UTD, Figure 8: flooding with $\mathrm{x}$ repetitions and flooding with $\mathrm{x}$ repetitions but stopped if a DENM is received from a vehicle behind. The graphs in Figure 7 show very similar DENM DD regardless of the number of repetitions and chosen dissemination strategy. From monitoring the average number of DENMs generated, however, (not shown in a figure) we see that the number of DENMs involved in the dissemination process is on average reduced from 100 for the model with 5 repetitions to merely 18 packets on average for the model where dissemination seized after DENM reception from behind. Furthermore, it should be noted that, for our parameter settings and the chosen transmission range and channel model, the DENM dissemination process is very often successful within one single DENM period. In other words, after a vehicle has received a DENM and re-broadcasted it once, no further periodic repetition is typically needed. However, facing the highly variable radio and network conditions of a real-world VANET setting, this might not always be the case and thus rebroadcasting a received DENM only once might not be sufficient to reach the entire platoon. In that case, the "until DENM reception from behind" method is able to provide the benefits of more repetitions, without introducing a high amount of unneeded packets into the network.

Figure 8 depicts the CAM UTD for different dissemination methods. For the cases with 100 extra vehicles, the gain from introducing as few DENM packets as possible into the network becomes obvious. A worst-case CAM UTD of approx. $200 \mathrm{~ms}$ (2 CAM periods) or less is achieved by only $70 \%$ of the simulated runs where flooding with 5 repetitions is used, compared to $96 \%$ of the cases with only 1 repetition or "flooding until DENM reception from behind". The corresponding percentage of CAMs that gain channel access at their first channel access attempt (not shown in a figure) are $20 \%$ and $25 \%$, for the two DENM dissemination strategies, respectively. A refinement of the dissemination model compared to simple flooding not only gains an advantage in DENM DD for unpredictable radio conditions but also leads to a significant improvement in CAM throughput as can be seen in the worst-case CAM UTD in Figure 8. It can be concluded that keeping the total number of packets to a minimum has a positive effect on the performance of both CAM and DENM.

\section{CONCLUSION}

In this paper, we conducted a delay study of co-existing periodic and event-triggered data traffic in a safety-critical platooning application. We evaluated the impact of the priority settings and send rates for CAM and DENM traffic, as well as suggested and evaluated a DENM dissemination strategy adapted to the platooning scenario. It should be noted that none of the studied test cases require changes to the IEEE 802.11p standard for intervehicle communication. Our study provides valuable input to the parameter selection for time-critical applications relying on the co-existence of the CAM and DENM traffic types on a common data channel, both for platooning applications in particular and other cooperative traffic safety applications in general.

Overall, it can be concluded that the choice of priority class, send rate and dissemination strategy influences the performance of both CAM and DENM traffic in the simulated platooning scenario. Key to a successful co-existence of periodic status messages and event-triggered warnings on a common channel is to not overload the medium with unnecessary data traffic. Controlling a platoon of tightly spaced vehicles moving at high speed puts high requirements on the up-to-dateness of periodic status messages. At the same time, in the case of an emergency, event-triggered warnings have to be spread within the platoon with minimal delay. For a platoon of 20 vehicles with 30 m antenna-to-antenna spacing and the assumption of a high overall channel utilization, we were able to achieve a platoon warning time of 6 ms or less for $80 \%$ of the simulated test runs while the entire platoon received a warning in $100 \%$ of the test runs after $10 \mathrm{~ms}$ or less. This number increases considerably when both CAM and DENM are assigned the same priority class, leading to platoon warning delays of up to $320 \mathrm{~ms}$. Even the CAM inter-arrival time is improved significantly by individual priority classes for periodic and event-triggered message types, regardless the fact that CAMs were given lower priority. An increased DENM send rate does not necessarily lead to a faster DENM dissemination and platoon warning time. We showed that the resulting bursts of DENMs following an eventdetection can in fact impair the successful channel access of both CAMs and DENMs due to an overloaded channel. To keep the number of DENMs involved in the warning process to a reasonable number we proposed a dissemination strategy that showed significant improvements in terms of CAM inter-arrival time while still maintaining platoon warning delays of $10 \mathrm{~ms}$ or below throughout the simulation.

\section{REFERENCES}

[1] Alam, A., Gattami, A., Johansson, K.H.: “An experimental study on the fuel reduction potential of heavy duty vehicle platooning" Proc. International Conference on Intelligent Transportation Systems, Madeira, Portugal, Sept 2010.

[2] IEEE 802.11p Part11: Wireless LAN Medium Access Control (MAC) and Physical Layer (PHY) specifications: Amendment 7: Wireless Access in Vehicular Environment, Jul. 2010. 
[3] ETSI ES 202 663: ITS; European profile standard for the physical and medium access layer of Intelligent Transport Systems operating in the $5 \mathrm{GHz}$ frequency band, Tech. Rep., 2010.

[4] ETSI TS 102 637-2: ITS; Vehicular Communications; Basic Set of Applications; Part 2: Specification on Cooperative Awareness Basic Services, Tech. Rep., 2010.

[5] ETSI TS 102 637-3: ITS; Vehicular Communications; Basic Set of Applications; Part 3: Specification of Decentralized Environmental Notification Basic Service, Tech. Rep., 2010.

[6] R. Reinders, M. van Eenennam, G. Karagiannis and G. Heijenk, "Contention window analysis for beaconing in VANETs", Proc. International Wireless Communications and Mobile Computing Conference (IWCMC), Istanbul, Turkey, Sept. 2011.

[7] M. Sepulcre and J. Gozálvez, "Optimizing adaptive transmission policies for wireless vehicular communications,"
Proc. IEEE Vehicular Technology Conference, Calgary, Canada, Sep. 2008.

[8] A. Böhm, M. Jonsson and E. Uhlemann, “Adaptive cooperative awareness messaging for enhanced overtaking assistance on rural roads,” Proc. IEEE Vehicular Technology Conference, San Francisco, CA, Sep. 2011, pp. 1-5.

[9] K. Bür and M. Kihl, "Evaluation of Selective Broadcast Algorithms for Safety Applications in Vehicular Ad Hoc Networks," Internatinal Journal of Vehicular Technology, 2001.

[10]A. Böhm, K. Lidström, M. Jonsson and T. Larsson, "Evaluating CALM M5-based vehicle-to-vehicle communication in various road settings through field trials," Proc. IEEE LCN Workshop on User Mobility and Vehicular Networks, Denver, CO, Oct. 2010. 